\title{
Novel Triterpenoids from the Fungus Ganoderma lucidum
}

\author{
Tsuyoshi Nishitoba, Kyoko Oda, Hiroji Sato \\ and Sadao SAKAMURA \\ Department of Agricultural Chemistry, Faculty of Agriculture, \\ Hokkaido University, Sapporo 060, Japan
}

Received August 11, 1987

The structures of eight novel triterpenoids, ganoderiol C (1), D (2), E (3), F (4), G (5), H (6), I (7), and ganolucidic acid E (8), isolated from the fruiting body of Ganoderma lucidum were determined by spectroscopic methods. In addition, the absolute configuration at $\mathrm{C}-23$ of ganolucidic acid D (9) was determined from the CD spectrum of its p-dimethylaminobenzoate derivative.

We have already reported a series of highly oxidized lanostane triterpenoids, some of them as bitter principles, from the fungus Ganoderma lucidum: ${ }^{1,2)}$ Recently, we further isolated the novel triterpene alcohols, ganoderiol $\mathrm{C}$ (1), D (2), E (3), F (4), G (5), H (6) and I (7), and triterpene acid, ganolucidic acid E (8), from the fruiting body of the fungus. In this paper, we describe their structural elucidation, and also mention the absolute configuration at C-23 of ganolucidic acid D (9) that was previously reported. ${ }^{3)}$


Ganoderiol C (1) was purified as its diacetate (1a), which gave a molecular ion peak at $m / z 602$ and was formulated to be $\mathrm{C}_{36} \mathrm{H}_{58} \mathrm{O}_{7}$ by HR-EI-MS. The IR spectrum of 1 a showed the presence of a hydroxyl group $\left(3460 \mathrm{~cm}^{-1}\right)$ and carbonyl groups $\left(1735,1705 \mathrm{~cm}^{-1}\right)$. The ${ }^{1} \mathrm{H}-\mathrm{NMR}$ signals at $\delta 4.90(1 \mathrm{H}, \mathrm{dd}), 4.14(1 \mathrm{H}$, d), $3.91(1 \mathrm{H}, \mathrm{d})$ and $1.21(3 \mathrm{H}, \mathrm{s})$ indicated that 1a had the same side chain with that of
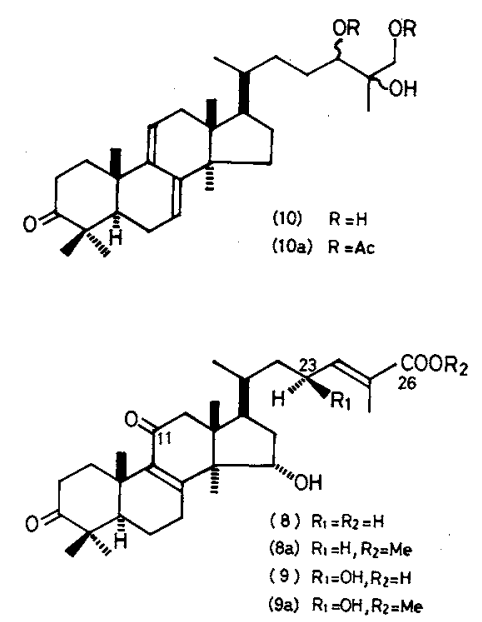
ganodermanontriol (10), ${ }^{5)}$ although 24 - and 26-hydroxyl groups were acetylated in 1a. The attachment of an $\alpha$-ethoxyl group at $\mathrm{C}-7$ was suggested by the signals at $\delta 3.77(1 \mathrm{H}$, br.s), $3.61(1 \mathrm{H}, \mathrm{dq}), 3.35(1 \mathrm{H}, \mathrm{dq})$ and $1.21(3 \mathrm{H}, \mathrm{dd})$, in analogy with the $7 \alpha$-methoxyl groups in the mycelial ganoderic acids. ${ }^{2)}$ This assignment was confirmed by converting 19 into 10 a with $\mathrm{H}_{2} \mathrm{SO}_{4}$. The free rotation about the bond between $\mathrm{C}-7$ and the attached oxygen is restricted by 1,3-diaxial-like interactions between the $7 \alpha$-ethoxyl group and $5 \alpha$-hydrogen and between the former and $14 \alpha$-methyl group. Therefore, the $7 \alpha$-ethoxyl group may be locat-

Table I. $\quad{ }^{13}$ C-NMR Data of 1a, 2, 3a, 4 and 6

$\left(67.8 \mathrm{MHz}\right.$ in $\mathrm{CDCl}_{3}$, except for 6 in $\left.\mathrm{C}_{5} \mathrm{D}_{5} \mathrm{~N}\right)$

\begin{tabular}{|c|c|c|c|c|c|}
\hline \multirow{2}{*}{ Carbon } & \multicolumn{5}{|c|}{$\delta$, ppm (Number of bonded $\mathrm{H}$ ) } \\
\hline & 1a & 2 & $3 a$ & 4 & 6 \\
\hline 1 & $35.2(2)$ & $35.4(2)$ & $34.6(2)$ & $36.1(2)$ & $35.2(2)$ \\
\hline 2 & $34.4(2)$ & $34.4(2)$ & $23.7(2)^{*}$ & $34.9(2)$ & $28.9(2)^{*}$ \\
\hline 3 & $217.6(0)$ & $214.6(0)$ & $79.6(1)$ & $216.8(0)$ & $77.1(1)$ \\
\hline 4 & $46.8(0)$ & $47.3(0)$ & $37.8(0)$ & $47.5(0)$ & $40.1(1)$ \\
\hline 5 & $45.0(1)$ & $49.1(1)$ & $49.0(1)$ & $50.8(1)$ & $49.6(1)$ \\
\hline 6 & $24.2(2)$ & $37.2(2)$ & $35.8(2)$ & $23.7(2)$ & $37.2(2)$ \\
\hline$i$ & $76.4(1)$ & $198.1(0)$ & $198.6(0)$ & $120.0(1)$ & $198.7(0)$ \\
\hline 8 & $135.5(0)$ & $139.6(0)$ & $139.0(0)$ & $142.9(0)$ & $138.9(0)$ \\
\hline 9 & $139.2(0)$ & $162.8(0)$ & $164.6(0)$ & $144.6(0)$ & $164.9(0)$ \\
\hline 10 & $37.8(0)$ & $39.5(0)$ & $39.7(0)$ & $37.3(0)$ & $39.5(0)$ \\
\hline 11 & $21.1(2)$ & $23.9(2)$ & $23.9(2)^{*}$ & $117.3(1)$ & $23.8(2)$ \\
\hline 12 & $30.3(2)$ & $30.2(2)$ & $30.2(2)$ & $37.9 .(2)$ & $30.5(2)$ \\
\hline 13 & $45.0(0)$ & $45.0(0)$ & $45.0(0)$ & $43.8(0)$ & $45.2(2)$ \\
\hline 14 & $50.0(0)$ & $47.8(0)$ & $47.8(0)$ & $50.4(0)$ & $48.2(0)$ \\
\hline 15 & $28.1(2)$ & $28.7(2)$ & $28.8(2)$ & $27.9(2)$ & $28.3(2)$ \\
\hline 16 & $31.1(2)$ & $31.9(2)$ & $32.0(2)$ & $31.5(2)$ & $32.7(2)$ \\
\hline 17 & $50.4(1)$ & $50.5(1)$ & $49.9(1)$ & $50.9(1)$ & $50.6(1)$ \\
\hline 18 & $16.2(3)^{*}$ & $16.0(3)$ & $15.8(3)$ & $15.8(3)$ & $16.1(3)^{*}$ \\
\hline 19 & $17.4(3)$ & $17.9(3)$ & $16.4(3)$ & $22.5(3)$ & $18.3(3)$ \\
\hline 20 & $36.7(1)$ & $36.6(1)$ & $36.2(1)$ & $36.1(1)$ & $37.3(1)$ \\
\hline 21 & $18.7(3)$ & $18.9(3)$ & $18.5(3)^{*}$ & $18.4(3)$ & $19.3(3)$ \\
\hline 22 & $32.7(2)$ & $33.6(2)$ & $36.5(2)$ & $36.7(2)$ & $34.5(2)$ \\
\hline 23 & $26.0(2)$ & $28.8(2)$ & $24.7(2)$ & $24.4(2)$ & $29.1(2)^{*}$ \\
\hline 24 & $74.8(1)$ & 79.1 (1) & $137.2(1)$ & $131.7(1)$ & $77.1(1)$ \\
\hline 25 & $73.3(0)$ & $73.9(0)$ & $128.8(0)$ & $136.8(0)$ & $74.8(0)$ \\
\hline 26 & $68.5(2)$ & $67.7(2)$ & $66.8(2)$ & $67.7(2)$ & $69.3(2)$ \\
\hline 27 & $20.2(3)$ & $20.9(3)$ & $59.9(2)$ & $60.2(2)$ & $20.1(3)$ \\
\hline 28 & $25.3(3)$ & $25.4(3)$ & $27.4(3)$ & $25.5(3)^{*}$ & $27.9(3)$ \\
\hline 29 & $21.4(3)$ & $21.4(3)$ & $21.2(3)$ & $22.1(3)$ & $16.0(3)^{*}$ \\
\hline 30 & $26.3(3)$ & $25.0(3)$ & $25.0(3)$ & $25.4(3)^{*}$ & $25.2(3)$ \\
\hline $7 \alpha-\mathrm{OCH}_{2} \mathrm{CH}_{3}$ & $64.0(2)$ & - & - & - & - \\
\hline $7 \alpha-\mathrm{OCH}_{2} \mathrm{CH}_{3}$ & $16.0(3)^{*}$ & - & - & - & - \\
\hline \multirow[t]{3}{*}{$-\mathrm{OCOCH}_{3}$} & $171.1(0)$ & - & $170.0(0)$ & - & - \\
\hline & $170.6(0)$ & - & $170.8(0)$ & - & - \\
\hline & - & - & $170.9(0)$ & - & - \\
\hline \multirow[t]{3}{*}{$-\mathrm{OCOCH}_{3}$} & $21.0(3)$ & 一 & $18.7(3)^{*}$ & - & - \\
\hline & $20.8(3)$ & - & $21.0(3)$ & - & - \\
\hline & - & - & $20.9(3)$ & - & - \\
\hline
\end{tabular}

* Assignments may be reversed. 
ed at the outside of the skeleton. In this situations, its methylene protons would not be magnetically equivalent and would show the unique coupling pattern in the ${ }^{1} \mathrm{H}-\mathrm{NMR}$ spectrum. The ${ }^{13} \mathrm{C}-\mathrm{NMR}$ spectrum of $\mathbf{1 a}$ (Table I) shows the presence of a carbonyl group at $\mathrm{C}-3$ and a double bond between C- 8 and C-9. On the basis of these observations, the depicted structure was concluded for 1a, and therefore, ganoderiol $\mathrm{C}$ was assigned to be $7 \alpha$-ethoxy24,25,26-trihydroxy-5 $\alpha$-lanost-8-en-3-one (1). It is not clear in this stage whether 1 was a natural product or an artifact.

Ganoderiol D (2) was obtained as a mixture with a small amount of ganoderiol E (3), which was acetylated to give the diacetate $\mathbf{2 a}$ $\left(\mathrm{C}_{34} \mathrm{H}_{52} \mathrm{O}_{7}\right)$ and triacetate $3 \mathbf{a}\left(\mathrm{C}_{36} \mathrm{H}_{54} \mathrm{O}_{7}\right)$. The ${ }^{1} \mathrm{H}-\mathrm{NMR}$ spectrum of $\mathbf{2 a}$ was similar to that of 1a, but showed no signals due to the $7 \alpha-$ ethoxyl group. The ${ }^{13} \mathrm{C}-\mathrm{NMR}$ spectrum of crude 2 showed clear data as listed in Table I, and indicates the presence of an $\alpha, \beta$-unsaturated carbonyl group $(\delta 198.1,162.8$ and 139.6), which was supported by the UV absorption maximum $(254 \mathrm{~nm})$ of $\mathbf{2 a}$. The position of the conjugated carbonyl group at $\mathrm{C}-7$ was ascertained from the the results of a ${ }^{1} \mathrm{H}$ NMR double resonance experiment on $\mathbf{2 a}$. The signals due to the $5 \alpha, 6 \alpha$ and $6 \beta$-protons were observed at ABX type at $\delta 2.14,2.33$ and 2.54 , respectively. From these data, the structure for 2a was determined, and ganoderiol D was deduced to be $24,25,26$-trihydroxy- $5 \alpha$ lanost-8-en-3,7-dione (2).

The ${ }^{1} \mathrm{H}-\mathrm{NMR}$ spectrum of $\mathbf{3 a}$ indicated the presence of an acetylated $3 \beta$-hydroxyl group $[\delta 4.51(1 \mathrm{H}, \mathrm{dd}, 3 \alpha-\mathrm{H})]$ and two acetylated allylic alcohols $[\delta 5.77(1 \mathrm{H}, \mathrm{t}), 4.66(2 \mathrm{H}, \mathrm{s})$ and $4.56(2 \mathrm{H}, \mathrm{s})]$ in the side chain by analogy with ganodermatriol (11). ${ }^{4)}$ The presence of an $\alpha, \beta$ unsaturated carbonyl group at C-7 was assumed from the ${ }^{13} \mathrm{C}-\mathrm{NMR}$ signals (198.6, 164.6 and 139.0; Table I) and the UV absorption maximum $(255 \mathrm{~nm})$. This assignment was supported by the ${ }^{1} \mathrm{H}-\mathrm{COSY}$ spectrum, in which the ABX-type coupling of the signals due to the $5 \alpha, 6 \alpha$ and $6 \beta$-protons was observed. The ${ }^{13} \mathrm{C}-\mathrm{NMR}$ signal of $\mathrm{C}-24$ was observed in a lower field $(\delta 137.2)$ than that of $\mathrm{C}-25$ $(\delta 128.8)$, in contrast with the data for ganodermanontriol $(\mathbf{1 0})^{5}$ ) and ganoderiol $F(4)$. This result may have been caused by acetylation of the hydroxyl groups at C-26 and C-27 in 3a. From these spectral evidences the represented structure was deduced for $\mathbf{3 a}$, and therefore, ganoderiol $\mathrm{E}$ was determined to be $3 \beta, 26,27$-trihydroxy-5 $\alpha$-lanosta-8,24-dien-7one (3).

Ganoderiol F (4), $\mathrm{C}_{30} \mathrm{H}_{46} \mathrm{O}_{3}$, showed similar ${ }^{1} \mathrm{H}$ - and ${ }^{13} \mathrm{C}-\mathrm{NMR}$ (Table I) spectra to those of ganodermatriol (11), but the presence of a carbonyl group at $\mathrm{C}-3$ in 4 , instead of the $\beta$ hydroxyl group in $\mathbf{1 1}$ was suggested by the ${ }^{13} \mathrm{C}-\mathrm{NMR}$ signal $(\delta 216.8)$. In the ${ }^{13} \mathrm{C}-\mathrm{NMR}$ spectra of 4 and 11, some differences were observed in the side chain moiety. This discrepancy is attributable to the solvents used $\left(\mathrm{CDCl}_{3}\right.$ for 4 and $\mathrm{C}_{5} \mathrm{D}_{5} \mathrm{~N}$ for 11). Consequently, the structure of ganoderiol $\mathrm{F}$ was defined to be 26,27-dihydroxy-5 $\alpha$-lanosta7,9(11),24-trien-3-one (4).

Ganoderiol $G(\mathbf{5})$ was formulated to be $\mathrm{C}_{31} \mathrm{H}_{52} \mathrm{O}_{5}$. Its ${ }^{1} \mathrm{H}-\mathrm{NMR}$ spectrum closely resembled that of 1 , but showed that the $7 \alpha-$ ethoxyl group in 1 was replaced by a $7 \alpha-$ methoxyl group $[\delta 3.67(1 \mathrm{H}$, br. s; $7 \beta-\mathrm{H})$, and $3.32(3 \mathrm{H}, \mathrm{s})]$. Therefore, ganoderiol $\mathrm{G}$ was concluded to be $24,25,26$-trihydroxy- $7 \alpha$ methoxy-5 $\alpha$-lanost-8-en-3-one (5).

Ganoderiol H (6), $\mathrm{C}_{30} \mathrm{H}_{50} \mathrm{O}_{5}$, showed a similar ${ }^{1} \mathrm{H}-\mathrm{NMR}$ spectrum to that of 2 , but the additional signal at $\delta 3.27(1 \mathrm{H}$, dd) indicated the presence of a $3 \beta$-hydroxyl group in 6 instead of the carbonyl group in 2 . The ${ }^{13} \mathrm{C}$ NMR data (Table I) of $\mathbf{6}$ also support this result. From these spectral evidences, ganoderiol $\mathrm{H}$ was elucidated to be $3 \beta, 24,25,26$ tetrahydroxy- $5 \alpha$-lanost-8-en-7-one (6).

The ${ }^{1} \mathrm{H}-\mathrm{NMR}$ spectrum of ganoderiol I (7), $\mathrm{C}_{31} \mathrm{H}_{50} \mathrm{O}_{5}$, was similar to that of ganoderiol $\mathrm{B}$ $(12),{ }^{\text {s) }}$ but the signals at $\delta 4.06(1 \mathrm{H}$, br. s) and $3.33(3 \mathrm{H}, \mathrm{s})$ suggested the presence of a $7 \alpha-$ methoxyl group. The former signal due to $7 \beta$ $H$ was deshielded by 0.39 ppm more than that of 5 , which was caused by the effect of the $15 \alpha-$ hydroxyl group. From these data, the structure 

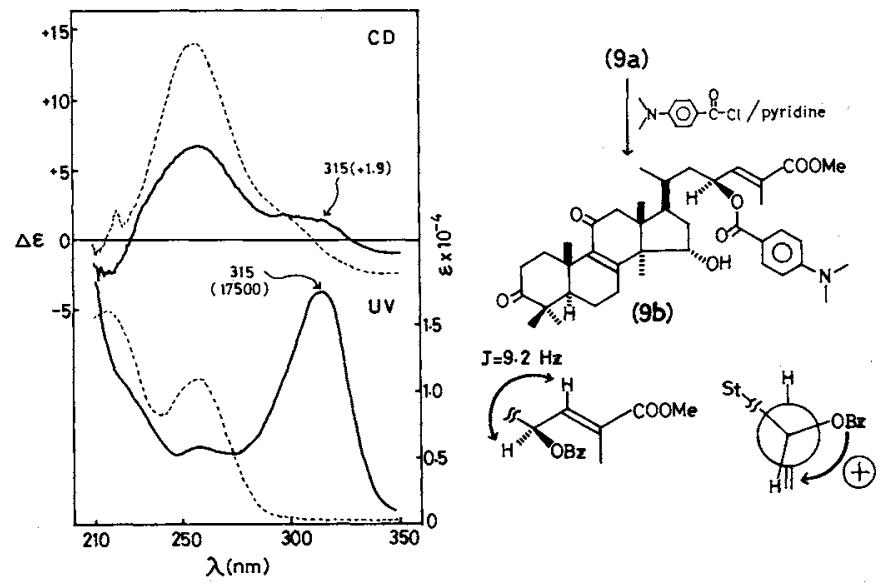

FIG. 1. CD and UV Spectra of $9 \mathrm{~b}(-)$ and $9 \mathrm{a}(----)$.

of ganoderiol I was assigned to be $15 \alpha, 26,27$ trihydroxy-5 $\alpha$-lanosta-8,24-dien-3-one (7).

Ganolucidic acid E (8) was purified as its methyl ester $\left(\mathbf{8 a}, \mathrm{C}_{31} \mathrm{H}_{46} \mathrm{O}_{5}\right)$. In the UV spectrum, 8a showed the presence of the $\alpha, \beta$ unsaturated carbonyl $(257 \mathrm{~nm})$ and carboxyl $(219 \mathrm{~nm})$ groups. The ${ }^{1} \mathrm{H}-\mathrm{NMR}$ spectrum of $\mathbf{8 a}$ closely resembled that of methyl ganolucidate $\mathrm{D}(9 \mathrm{a}),{ }^{3)}$ but with the hydroxyl group absent at $\mathrm{C}-23$. The olefinic proton at C-24 was observed in a lower field $[\delta 6.73(1 \mathrm{H}, \mathrm{tq})]$ than that $[\delta 6.59(1 \mathrm{H}, \mathrm{dq})]$ of $9 \mathbf{a}$. From these observations, the depicted structure was proposed for $\mathbf{8 a}$, and the original ganolucidic acid E was established to be $15 \alpha$-hydroxy-3,11dioxo-5 $\alpha$-lanosta-8,24E-dien-26-oic acid (8).

Biogenetically, ganolucidic acid D (9) may be produced from ganolucidic acid $\mathrm{E}(\mathbf{8})$ by allylic oxidation at $\mathrm{C}-23$, and be further converted to a series of ganoderic acids. "So it is important to resolve the stereochemistry of the hydroxyl group at C-23 in 9. 9a was converted to its mono- $p$-dimethylaminobenzoate derivative $\left(9 \mathrm{~b}, \mathrm{C}_{40} \mathrm{H}_{55} \mathrm{NO}_{7}\right)$. The $\mathrm{UV}$ and $\mathrm{CD}$ spectra of $\mathbf{9 b}$ are presented in Fig. 1 with those of $9 \mathbf{a}$, 9b showing a positive Cotton effect at $315 \mathrm{~nm}$ $(\Delta \varepsilon+1.9)$ caused by mutual interaction between the conjugated carboxyl group and the $p$-dimethylaminobenzoate chromophore. Taking account of this result and the ${ }^{1} \mathrm{H}-\mathrm{NMR}$ coupling constant $(9.2 \mathrm{~Hz})$ between $23-\mathrm{H}$ and $24-\mathrm{H}$ in $9 \mathbf{b}$, the positive chirality between the two chromophores was designated as shown in Fig. $1{ }^{6)}$ Therefore, the absolute configuration at $\mathrm{C}-23$ in $9 \mathrm{~b}$ was unequivocally defined to be $S$. Since no stereochemical inversion existed in the converting process from 9 into $9 \mathbf{b}$, the absolute configuration at C-23 in 9 was also assigned to be $S$.

\section{EXPERIMENTAL}

General. Melting points (mp) were measured with a Yanaco micro-melting point apparatus and are uncorrected. Optical rotations were measured on a JASCO DIP4 instrument, and CD spectra were measured with $\mathrm{JASCO}$ J-20 instrument. IR and UV spectra were obtained with a Hitachi 285 spectrometer and Hitachi EPS-3T spectrophotometer, respectively. ${ }^{1} \mathrm{H}$ - and ${ }^{13} \mathrm{C}$-NMR spectra were recorded on a JEOL FX-270 spectrometer. Column chromatography was carried out with Wakogel C-200 (Wako), Kieselgel $60(230 \sim 400$ mesh, Merck) and a Lobar column (RP-18, Merck). PTLC was performed on pre-coated Kieselgel $60 \mathrm{~F}_{254}(0.5 \mathrm{~mm}$ thickness, Merck) and RP-18 $\mathrm{F}_{254} \mathrm{~s}$ (Merck). TLC was done on pre-coated Kieselgel 60 $F_{254}$ plates $(0.2 \mathrm{~mm}$ thickness, Merck).

Extraction and isolation. The neutral fraction $(217 \mathrm{~g})$ obtained from the EtOH extract of G. lucidum (a mixture of two strains ${ }^{7)}$ ) was separated into three fractions (Fr. I, Fr. II and Fr. III) as previously reported. ${ }^{5)}$ Fr. I was further separated by silica gel and Lobar column chromatographies into several fractions (Frs. D, K, M and P). Frs. $\mathrm{D}$ and $\mathrm{K}$ were acetylated $\left(\mathrm{Ac}_{2} \mathrm{O}\right.$ /pyridine) and subjected to silica gel column chromatography to give com- 
pounds 1a $(107 \mathrm{mg})$ and $2 \mathbf{a}(50.5 \mathrm{mg})$, and $3 \mathbf{a}(6.9 \mathrm{mg})$, respectively, Compound 4 (56 $\mathrm{mg})$ was crystallized $\left(\mathrm{CHCl}_{3}-\mathrm{MeOH}\right)$ from Fr. K. Compound $8 \mathbf{a}(3 \mathrm{mg})$ was obtained by PTLC of Fr. P after methylation with $\mathrm{CH}_{2} \mathrm{~N}_{2}$. On the other hand, Fr. III was rechromatographed four times on a silica gel column to give Fr. U, which was further purified by the combination of column chromatography, PTLC and crystallization to yield compounds $5(3 \mathrm{mg}), 6(15 \mathrm{mg})$ and $7(3 \mathrm{mg})$.

24,26-Di-O-acetyl ganoderiol C (1a). Colorless syrup,

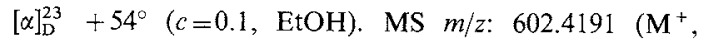
$\left.\mathrm{C}_{36} \mathrm{H}_{58} \mathrm{O}_{7}, 2 \%\right), 587(5), 556\left(\mathrm{M}^{+}-\mathrm{C}_{2} \mathrm{H}_{5} \mathrm{OH}, 8\right), 496(8)$, $309(46), 43(100)$. IR $v_{\max }(\mathrm{film}) \mathrm{cm}^{-1}: 3460,2960,2870$, 1735, 1705. NMR $\delta_{\mathrm{H}}\left(\mathrm{CDCl}_{3}\right): 4.90(1 \mathrm{H}, \mathrm{dd}, J=10.3,2.6$; $24-\mathrm{H}), 4.14(1 \mathrm{H}, \mathrm{d}, J=11.4 ; 26-\mathrm{H}), 3.91(1 \mathrm{H}, \mathrm{d}, J=11.4$; $26-\mathrm{H}), 3.77(.1 \mathrm{H}$, br.s; $7 \beta-\mathrm{H}), 3.61(1 \mathrm{H}, \mathrm{dq}, J=8.8,7.0 ; 7 \alpha-$ $\left.\mathrm{OCH}_{2} \mathrm{CH}_{3}\right), 3.35\left(1 \mathrm{H}, \mathrm{dq}, J=8.8,7.0 ; 7 \alpha-\mathrm{OCH}_{2} \mathrm{CH}_{3}\right)$, $1.21\left(3 \mathrm{H}, \mathrm{dd}, J=7.0,7.0 ; 7 \alpha-\mathrm{OCH}_{2} \mathrm{CH}_{3}\right), 1.21 \overline{(3 \mathrm{H}}, \mathrm{s} ; 27-$ $\mathrm{H}), 1.11(3 \mathrm{H}, \mathrm{s} ; 28-\mathrm{H}), 1.08(3 \mathrm{H}, \mathrm{s} ; 29-\mathrm{H}), 1.06(3 \mathrm{H}, \mathrm{s} ; 19$ or $30-\mathrm{H}), 1.05(3 \mathrm{H}, \mathrm{s} ; 19$ or $30-\mathrm{H}), 0.90(3 \mathrm{H}, \mathrm{d}, J=6.6 ; 2 \mathrm{I}$. $\mathrm{H}), 0.62(3 \mathrm{H}, \mathrm{s} ; 18-\mathrm{H}), 2.11\left(3 \mathrm{H}, \mathrm{s} ; \mathrm{OCOCH}_{3}\right), 2.07(3 \mathrm{H}, \mathrm{s}$; $\mathrm{OCOCH}_{3}$ ). Alkaline treatment of la gave the original ganoderiol C (1), which was confirmed on TLC.

Acid treatment of 1a. 1a $(6.2 \mathrm{mg})$ was dissolved in acetone $(2.5 \mathrm{ml})$, and to the solution $5 \mathrm{~N}-\mathrm{H}_{2} \mathrm{SO}_{4}$ was added at $0^{\circ} \mathrm{C}$. The reaction mixture was stirred at room temperature for thirty minutes. After removing the acetone in vacuo, the solution was diluted with water and extracted with $\mathrm{CHCl}_{3}$. The organic layer was dried with $\mathrm{Na}_{2} \mathrm{SO}_{4}$ and then concentrated to give the product (10a), which was purified by PTLC (5.5 mg, yield). MS $m / z: 556.3793\left(\mathrm{M}^{+}\right.$, $\mathrm{C}_{34} \mathrm{H}_{52} \mathrm{O}_{6}, 3 \%$ ), $538(5), 496(5), 309(50), 269(26), 55(36)$, $43(100) . \mathrm{NMR} \delta_{\mathrm{H}}\left(\mathrm{CDCl}_{3}\right): 5.50(1 \mathrm{H}, \mathrm{d}, J=6.2 ; 7-\mathrm{H})$, $5.38(1 \mathrm{H}, \mathrm{d}, J=5.5 ; 11-\mathrm{H}), 4.90(1 \mathrm{H}, \mathrm{dd}, J=10.0,2.7 ; 24-$ $\mathrm{H}), 4.14(1 \mathrm{H}, \mathrm{d}, J=11.4 ; 26-\mathrm{H}), 3.91(1 \mathrm{H}, \mathrm{d}, J=11.4 ; 26-$ H), $2.77(1 \mathrm{H}$, ddd, $J=14.8,14.8,6.0 ; 2 \beta-\mathrm{H}), 1.21(3 \mathrm{H}, \mathrm{s}$; $27-\mathrm{H}), 1.20(3 \mathrm{H}, \mathrm{s} ; 19-\mathrm{H}), 1.13(3 \mathrm{H}, \mathrm{s} ; 28-\mathrm{H}), 1.09(3 \mathrm{H}, \mathrm{s}$; $29-\mathrm{H}), 0.91(3 \mathrm{H}, \mathrm{d}, J=6.2 ; 21-\mathrm{H}), 0.87(3 \mathrm{H}, \mathrm{s} ; 30-\mathrm{H}), 0.58$ $(3 \mathrm{H}, \mathrm{s} ; 18-\mathrm{H}), 2.11\left(3 \mathrm{H}, \mathrm{s} ; \mathrm{OCOCH}_{3}\right), 2.08(3 \mathrm{H}, \mathrm{s}$; $\mathrm{OCOCH}_{3}$ )

24,26-Di-O-acetyl ganoderiol D (2a). Colorless syrup, $[\alpha]_{\mathrm{D}}^{21}+8^{\circ}(c=0.1, \mathrm{MeOH})$. MS $m / z: 572.3639\left(\mathrm{M}^{+}\right.$, $\mathrm{C}_{34} \mathrm{H}_{52} \mathrm{O}_{7}, 6 \%$ ), $557(6), 339(15), 327(13), 69(65), 57(87)$, 43 (100). IR $v_{\max }\left(\right.$ film) $\mathrm{cm}^{-1}: 3440,2950,2860,1730,1705$, 1655, 1575. UV $\hat{n}_{\max }(\mathrm{MeOH}) \mathrm{nm}(\varepsilon): 254$ (9730). NMR $\delta_{\mathrm{H}}$ $\left(\mathrm{CDCl}_{3}\right): 4.90(1 \mathrm{H}, \mathrm{dd}, J=9.9,2.6 ; 24-\mathrm{H}), 4.13(1 \mathrm{H}, \mathrm{d}, J=$ $11.4 ; 26-\mathrm{H}), 3.90(1 \mathrm{H}, \mathrm{d}, J=11.4 ; 26-\mathrm{H}), 2.70(1 \mathrm{H}, \mathrm{ddd}$, $J=15.8,12.1,7.0 ; 2 \beta-\mathrm{H}), 2.54(1 \mathrm{H}, \mathrm{dd}, J=16.0,14.5 ; 6 \beta-$ $\mathrm{H}), 2.46(1 \mathrm{H}, \mathrm{ddd}, J=15.8,5.9,3.7 ; 2 \alpha-\mathrm{H}), 2.33(1 \mathrm{H}$, dd, $J=16.0,3.3 ; 6 \alpha-\mathrm{H}), 2.14(1 \mathrm{H}, \mathrm{dd}, J=14.5,3.3 ; 5 \alpha-\mathrm{H}), 1.33$ $(3 \mathrm{H}, \mathrm{s} ; 30-\mathrm{H}), 1.20(3 \mathrm{H}, \mathrm{s} ; 27-\mathrm{H}), 1.12(3 \mathrm{H}, \mathrm{s} ; 28-\mathrm{H}), 1.10$ $(3 \mathrm{H}, \mathrm{s} ; 29-\mathrm{H}), 0.93(3 \mathrm{H}, \mathrm{s} ; 19-\mathrm{H}), 0.92(3 \mathrm{H}, \mathrm{d}, J=5.9 ; 21-$ $\mathrm{H}), 0.67(3 \mathrm{H}, \mathrm{s} ; 18-\mathrm{H})$.
3,26,27-Tri-O-acetyl ganoderiol E (3a). Colorless syrup, $[\alpha]_{\mathrm{D}}^{21}+18^{\circ}(c=0.1, \mathrm{MeOH})$. MS $m / z: 598.3836\left(\mathrm{M}^{+}\right.$, $\mathrm{C}_{36} \mathrm{H}_{54} \mathrm{O}_{7}, 13 \%$ ), 583 (11), 538 (15), 463 (28), 369 (23), 121 (42), 43 (100). IR $v_{\max }\left(\right.$ film) $\mathrm{cm}^{-1}: 3000,2940,2860,1720$, 1650, 1570. UV $\lambda_{\max }(\mathrm{MeOH}) \mathrm{nm}(\varepsilon): 255$ (9160). NMR $\delta_{\mathrm{H}}$ $\left(\mathrm{CDCl}_{3}\right): 5.77(1 \mathrm{H}, \mathrm{t}, J=7.3 ; 24-\mathrm{H}), 4.66(2 \mathrm{H}, \mathrm{s} ; 27-\mathrm{H})$, $4.56(2 \mathrm{H}, \mathrm{s} ; 26-\mathrm{H}), 4.51(1 \mathrm{H}, \mathrm{dd}, J=11.4,4.4 ; 3-\mathrm{H}), 2.46$ $(1 \mathrm{H}, \mathrm{dd}, J=16.0,12.0 ; 6 \beta-\mathrm{H}), 2.38(1 \mathrm{H}, \mathrm{dd}, J=16.0,5.0$; $6 \alpha-\mathrm{H}), 1.19(3 \mathrm{H}, \mathrm{s} ; 30-\mathrm{H}), 0.95(3 \mathrm{H}, \mathrm{s} ; 19-\mathrm{H}), 0.93(3 \mathrm{H}, \mathrm{d}$, $J=5.9 ; 21-\mathrm{H}), 0.91(3 \mathrm{H}, \mathrm{s} ; 28-\mathrm{H}), 0.89(3 \mathrm{H}, \mathrm{s} ; 29-\mathrm{H}), 0.65$ $(3 \mathrm{H}, \mathrm{s} ; 18-\mathrm{H}), 2.06\left(9 \mathrm{H}, \mathrm{s} ; \mathrm{OCOCH}_{3} \times 3\right)$.

Ganoderiol $F(4) .4$ was obtained as pale yellow plates (from $\mathrm{CHCl}_{3}-\mathrm{MeOH}$ ), mp $116 \sim 120^{\circ} \mathrm{C},[\alpha]_{\mathrm{D}}^{21}+42^{\circ}(c=$ $0.1, \mathrm{MeOH}) . \mathrm{MS} m / z: 454.3430\left(\mathrm{M}^{+}, \mathrm{C}_{30} \mathrm{H}_{46} \mathrm{O}_{3}, 4 \%\right), 436$ (6), $309(100), 69(24), 55(40), 41$ (31). IR $v_{\max }\left(\right.$ film) $\mathrm{cm}^{-1}$ : 3350, 2900, 2850, 1690. UV $\hat{\lambda}_{\max }(\mathrm{MeOH}) \mathrm{nm}(\varepsilon): 237$ (16600), $245(19200), 253(12500)$. NMR $\delta_{\mathrm{H}}\left(\mathrm{CDCl}_{3}\right): 5.56$ $(1 \mathrm{H}, \mathrm{t} ; J=7.3 ; 24-\mathrm{H}), 5.51(1 \mathrm{H}, \mathrm{d}, J=6.9 ; 7-\mathrm{H}), 5.39(1 \mathrm{H}$, d, $J=5.9 ; 11-\mathrm{H}), 4.33(2 \mathrm{H}, \mathrm{s} ; 27-\mathrm{H}), 4.22(2 \mathrm{H}, \mathrm{s} ; 26-\mathrm{H})$, $2.78(1 \mathrm{H}, \mathrm{ddd}, J=14.7,14.7,5.5 ; 2 \beta-\mathrm{H}), 1.20(3 \mathrm{H}, \mathrm{s} ; 19-$ H), $1.13(3 \mathrm{H}, \mathrm{s} ; 28-\mathrm{H}), 1.09(3 \mathrm{H}, \mathrm{s} ; 29-\mathrm{H}), 0.92(3 \mathrm{H}, \mathrm{d}, J=$ $6.6 ; 21-\mathrm{H}), 0.88(3 \mathrm{H}, \mathrm{s} ; 30-\mathrm{H}), 0.59(3 \mathrm{H}, \mathrm{s} ; 18-\mathrm{H})$.

Ganoderiol $G(\mathbf{5})$. Colorless syrup, $[\alpha]_{\mathrm{D}}^{24}+34^{\circ}(c=0.1$, $\mathrm{MeOH})$. MS m/z: $504.3860\left(\mathrm{M}^{+}, \mathrm{C}_{31} \mathrm{H}_{52} \mathrm{O}_{5}, 22 \%\right), 489$ (43), $472(17), 457(19), 366(64), 95(55), 69(62), 55(78)$, 43 (100). IR $v_{\max }(\mathrm{film}) \mathrm{cm}^{-1}: 3380,2920,2870,1700$. NMR $\delta_{\mathrm{H}}\left(\mathrm{CDCl}_{3}\right): 3.84(1 \mathrm{H}, \mathrm{d}, J=11.4 ; 26-\mathrm{H}), 3.67(1 \mathrm{H}$, br. $\mathrm{s} ; \beta-\mathrm{H}), 3.49(1 \mathrm{H}, \mathrm{d}, J=11.0 ; 26-\mathrm{H}), 3.47(1 \mathrm{H}, \mathrm{t}, J=$ $8.0 ; 24-\mathrm{H}), 3.32\left(3 \mathrm{H}, \mathrm{s} ; 7 \alpha-\mathrm{OCH}_{3}\right), 1.26(3 \mathrm{H}, \mathrm{s} ; 27-\mathrm{H}), 1.12$ $(3 \mathrm{H}, \mathrm{s} ; 28-\mathrm{H}), 1.09(3 \mathrm{H}, \mathrm{s} ; 29-\mathrm{H}), 1.05(3 \mathrm{H}, \mathrm{s} ; 30-\mathrm{H}), 1.05$ $(3 \mathrm{H}, \mathrm{s} ; 19-\mathrm{H}), 0.90(3 \mathrm{H}, \mathrm{d}, J=8.8 ; 21-\mathrm{H}), 0.64(3 \mathrm{H}, \mathrm{s} ; 18-$ $\mathrm{H})$.

Ganoderiol $H(6) .6$ was obtained as colorless needles, mp $200 \sim 201.5^{\circ} \mathrm{C},[\alpha]_{\mathrm{D}}^{24}+22^{\circ}(c=0.1, \mathrm{MeOH})$. MS $m / z$ : $490.3625\left(\mathrm{M}^{+}, \mathrm{C}_{30} \mathrm{H}_{50} \mathrm{O}_{5}, 30 \%\right), 475(47), 415(46), 381$ (35), $329(35), 121(74), 69(59), 55(68), 43(100)$. IR $v_{\max }$ (film) $\mathrm{cm}^{-1}: 3380,2950,2860,1640 . \mathrm{UV} \hat{\lambda}_{\max }(\mathrm{MeOH}) \mathrm{nm}$ (ع): $255(9170)$. NMR $\delta_{\mathrm{H}}\left(\mathrm{CDCl}_{3}\right): 3.82(1 \mathrm{H}, \mathrm{d}, J=11.4$; $26-\mathrm{H}), 3.46(1 \mathrm{H}, \mathrm{d}, J=11.4 ; 26-\mathrm{H}), 3.43(1 \mathrm{H}, \mathrm{dd}, J=10.0$, $1.5 ; 24-\mathrm{H}), 3.27(1 \mathrm{H}, \mathrm{dd}, J=11.2,4.9 ; 3 \alpha-\mathrm{H}), 2.46(1 \mathrm{H}$, dd, $J=16.5,11.7 ; 6 \beta-\mathrm{H}), 2.38(1 \mathrm{H}, \mathrm{dd}, J=16.5,6.2 ; 6 \alpha-\mathrm{H})$, $1.17(3 \mathrm{H}, \mathrm{s} ; 30-\mathrm{H}), 1.11(3 \mathrm{H}, \mathrm{s} ; 27-\mathrm{H}), 1.00(3 \mathrm{H}, \mathrm{s} ; 28-\mathrm{H})$, $0.93(3 \mathrm{H}, \mathrm{d}, J=7.7 ; 21-\mathrm{H}), 0.92(3 \mathrm{H}, \mathrm{s} ; 19-\mathrm{H}), 0.88(3 \mathrm{H}, \mathrm{s}$; $29-\mathrm{H}), 0.66(3 \mathrm{H}, \mathrm{s} ; 18-\mathrm{H})$.

Ganoderiol $I(7)$. Colorless syrup, $[\alpha]_{D}^{22}+53^{\circ}(c=0.1$, $\mathrm{MeOH})$. MS $m / z: 502.3675\left(\mathrm{M}^{+}, \mathrm{C}_{31} \mathrm{H}_{50} \mathrm{O}_{5}, 3 \%\right), 470(7)$, $452(2), 349(59), 325(45), 175(66), 69(60), 55(99), 43$ (100). IR $v_{\max }\left(\right.$ film) $\mathrm{cm}^{-1}: 3380,2920,2870,1700 . \mathrm{NMR}$ $\delta_{\mathrm{H}}\left(\mathrm{CDCl}_{3}\right): 5.55(1 \mathrm{H}, \mathrm{t}, J=7.3 ; 24-\mathrm{H}), 4.35(1 \mathrm{H}, \mathrm{t}, J=8.8$; $15 \beta-\mathrm{H}), 4.33(2 \mathrm{H}, \mathrm{s} ; 27-\mathrm{H}), 4.22(2 \mathrm{H}, \mathrm{s} ; 26-\mathrm{H}), 4.06(1 \mathrm{H}$, br.s; $7 \beta-\mathrm{H}), 3.33\left(3 \mathrm{H}, \mathrm{s} ; 7 \alpha-\mathrm{OCH}_{3}\right), 2.58(\mathrm{lH}$, ddd, $J=$ $15.9,10.6,7.3 ; 2 \beta-\mathrm{H}), 2.46$ (1H, ddd, $J=15.9,7.3,3.8 ; 2 \alpha-$ 
H), $1.13(3 \mathrm{H}, \mathrm{s} ; 28-\mathrm{H}), 1.10(3 \mathrm{H}, \mathrm{s} ; 29$ or $30-\mathrm{H}), 1.08(3 \mathrm{H}$, $\mathrm{s} ; 29$ or $30-\mathrm{H}), 1.02(3 \mathrm{H}, \mathrm{s} ; 19-\mathrm{H}), 0.89(3 \mathrm{H}, \mathrm{d}, J=6.6 ; 21$ $\mathrm{H}), 0.66(3 \mathrm{H}, \mathrm{s} ; 18-\mathrm{H})$.

Methyl ganolucidate $E(\mathbf{8 a})$. Colorless syrup, $[\alpha]_{\mathrm{D}}^{24}+154^{\circ}$ $(c=0.1, \mathrm{MeOH})$. MS $m / z: 498.3335\left(\mathrm{M}^{+}, \mathrm{C}_{31} \mathrm{H}_{46} \mathrm{O}_{5}\right.$, $22 \%), 470(18), 401(100), 287(69), 95(34), 69(28), 55(36)$, 41 (29). IR $v_{\max }\left(\right.$ film) $\mathrm{cm}^{-1}: 3450,2940,2920,2870,1700$, 1635, 1570. UV $i_{\max }(\mathrm{MeOH}) \mathrm{nm}(\varepsilon): 219(12100), 257$ (7080). NMR $\delta_{\mathrm{H}}\left(\mathrm{CDCl}_{3}\right): 6.73(1 \mathrm{H}, \mathrm{tq}, J=7.5,1.5 ; 24-\mathrm{H})$, $4.39(1 \mathrm{H}, \mathrm{dd}, J=9.2,5.7 ; 15 \beta-\mathrm{H}), 3.01$ ( $1 \mathrm{H}$, ddd, $J=13.9$, $8.2,5.6 ; 1 \beta-\mathrm{H}), 2.72(1 \mathrm{H}, \mathrm{d}, J=17.2 ; 12 \alpha-\mathrm{H}), 2.45(1 \mathrm{H}, \mathrm{d}$, $J=16.9 ; 12 \beta-\mathrm{H}), 1.83(3 \mathrm{H}, \mathrm{s} ; 27-\mathrm{H}), 1.18(3 \mathrm{H}, \mathrm{s} ; 30-\mathrm{H})$, $1.13(3 \mathrm{H}, \mathrm{s} ; 19$ or $28-\mathrm{H}), 1.12(3 \mathrm{H}, \mathrm{s} ; 19$ or $28-\mathrm{H}), 1.08(3 \mathrm{H}$, $\mathrm{s} ; 29-\mathrm{H}), 0.89(3 \mathrm{H}, \mathrm{s} ; 18-\mathrm{H}), 0.89(3 \mathrm{H}, \mathrm{d}, J=6.2 ; 21-\mathrm{H})$, $3.74\left(3 \mathrm{H}, \mathrm{s} ; \mathrm{COOCH}_{3}\right)$.

Preparation of compound 9 b. Methyl ganolucidate D (9a, $2.9 \mathrm{mg}$ ) and $p$-dimethylaminobenzoyl chloride $(5 \mathrm{mg}$ ) were dissolved in anhydrous pyridine $(0.1 \mathrm{ml})$ and refluxed under nitrogen with stirring. Two hours later, the reaction was terminated by adding ice-cold water to the reaction mixture, which was then extracted with EtOAc. After washing with sat. aq. $\mathrm{NH}_{4} \mathrm{Cl}$ and sat. aq. $\mathrm{NaCl}$, the organic layer was dried with $\mathrm{Na}_{2} \mathrm{SO}_{4}$ and concentrated. The products were purified by PTLC to yield the 23- $O$ monobenzoate $\left(9 \mathrm{~b}, \mathrm{C}_{40} \mathrm{H}_{55} \mathrm{NO}_{7}, 0.4 \mathrm{mg}\right)$ and $15 \alpha, 23-O$ dibenzoate (9c, $\mathrm{C}_{49} \mathrm{H}_{64} \mathrm{~N}_{2} \mathrm{O}_{8}, 1.5 \mathrm{mg}$ ). The physicochemical properties for $\mathbf{9 b}$ are as follows: MS m/z: 661.3966 $\left(\mathrm{M}^{+}, 6.6 \%\right), 165(100), 148(40), 125(23), 95(16), 69(22)$, $55(28), 44(44)$. UV $\lambda_{\max }(\mathrm{EtOH}) \mathrm{nm}(\varepsilon): 315(17500), 258$ (5780). CD $\lambda(c=0.01$, EtOH) nm $(\Delta \varepsilon): 328(0), 315$ $(+1.9), 256(+6.6), 227(0) . \mathrm{NMR} \delta_{\mathrm{H}}\left(\mathrm{CDCl}_{3}\right): 7.88(2 \mathrm{H}$, $\mathrm{d}, J=9.0), 6.64(2 \mathrm{H}, \mathrm{d}, J=9.0), 6.64(1 \mathrm{H}$, overlapped; $24-$ $\mathrm{H}), 5.80(1 \mathrm{H}, \mathrm{m} ; 23-\mathrm{H}), 4.37(1 \mathrm{H}, \mathrm{m} ; 15 \beta-\mathrm{H}), 3.75(3 \mathrm{H}, \mathrm{s}$; $\left.\mathrm{COOCH}_{3}\right), 3.04(6 \mathrm{H}, \mathrm{s}), 2.70(1 \mathrm{H}, \mathrm{d}, J=17.4 ; 12 \alpha-\mathrm{H}), 2.43$ $(\mathrm{IH}, \mathrm{d}, J=17.4 ; 12 \beta-\mathrm{H}), 2.00(3 \mathrm{H}, \mathrm{s} ; 27-\mathrm{H}), 1.26(3 \mathrm{H}, \mathrm{s}$;
$30-\mathrm{H}), 1.12(6 \mathrm{H}, \mathrm{s} ; 19$ and $29-\mathrm{H}), 1.08(3 \mathrm{H}, \mathrm{s} ; 28-\mathrm{H}), 0.99$ $(3 \mathrm{H}, \mathrm{d}, J=6.6 ; 21-\mathrm{H}), 0.86(3 \mathrm{H}, \mathrm{s} ; 18-\mathrm{H}) . \mathrm{NMR} \delta_{\mathrm{H}}$ $\left(\mathrm{C}_{5} \mathrm{D}_{5} \mathrm{~N}\right): 8.25(2 \mathrm{H}, \mathrm{d}, J=9.2), 7.00(1 \mathrm{H}, \mathrm{dq}, J=9.2,1.5$; $24-\mathrm{H}), 6.74(2 \mathrm{H}, \mathrm{d}, J=9.2), 6.15(1 \mathrm{H}, \mathrm{m} ; 23-\mathrm{H}), 4.64(1 \mathrm{H}$, $\mathrm{m}), 3.65(3 \mathrm{H}, \mathrm{s}), 2.92(\mathrm{lH}, \mathrm{d}, J=17.2), 2.80(6 \mathrm{H}, \mathrm{s}), 2.64$ $(1 \mathrm{H}, \mathrm{d}, J=17.2), 2.17(1 \mathrm{H}, \mathrm{d}, J=1.5 ; 27-\mathrm{H}), 1.48(3 \mathrm{H}, \mathrm{s})$, $1.25(3 \mathrm{H}, \mathrm{s}), 1.12(6 \mathrm{H}, \mathrm{s}), 1.09(3 \mathrm{H}, \mathrm{d}, J=6.6), 0.94(3 \mathrm{H}, \mathrm{s})$.

Acknowledgments. We are grateful to Ms. Y. Misu ( ${ }^{1} \mathrm{H}-$ and ${ }^{13} \mathrm{C}-\mathrm{NMR}$ ), Mr. K. Watanabe (MS), Ms. Y. Atsuta (MS) and their associates at Hokkaido University for spectral measurements.

\section{REFERENCES}

1) T. Nishitoba, H. Sato and S. Sakamura, Phytochemistry, 26, 1777 (1987); and references cited therein.

2) T. Nishitoba, H. Sato, S. Shirasu and S. Sakamura, Agric. Biol. Chem., 51, 619 (1987); T. Nishitoba, H. Sato and S. Sakamura, Agric. Biol. Chem., 51, 1149 (1987).

3) T. Nishitoba, H. Sato and S. Sakamura, Agric. Biol. Chem., 50, 809 (1986).

4) M. Arisawa, A. Fujita, M. Saga, H. Fukumura, T. Hayashi, M. Shimizu and N. Morita, J. Natl. Prod., 49, 621 (1986).

5) H. Sato, T. Nishitoba, S. Shirasu, K. Oda and S. Sakamura, Agric. Biol. Chem., 50, 2887 (1986).

6) N. Harada, J. Iwabuchi, Y. Yokota, H. Uda, M. Ochi, N. C. Gonnella, K. Nakanishi, V. S. Martin and K. B. Sharpless, Symposium Papers, 25th Symposium on the Chemistry of Natural Products, Tokyo, October, 1982, p. 39.

7) T. Nishitoba, H. Sato, S. Shirasu and S. Sakamura, Agric. Biol. Chem., 50, 2151 (1986). 